\title{
Numerical Simulations of the Equations of Particle Motion in the Gas Flow
}

\author{
Kelong Zheng ${ }^{1^{*}}$, Liuxiang Zhang ${ }^{2}$, Haiyan Chen ${ }^{2}$ \\ ${ }^{1}$ School of Science, Southwest University of Science and Technology, Mianyang, China \\ ${ }^{2}$ School of Environment and Resource, Southwest University of Science and Technology, Mianyang, China \\ Email: zhengkelong@swust.edu.cn
}

Received 2013

\begin{abstract}
Under the assumption of considering the gravity and without gravity, two different acceleration models to describe particle' motion in the gas flow are formulated, respectively. The corresponding numerical simulations of these models do not only show the trend of the velocity of the particle in different density and particle diameter sizes, but also the relationship between the maximum particle velocity and its diameter size.
\end{abstract}

Keywords: Numerical Simulation; Particle Motion; Acceleration; Jet Grinding

\section{Introduction}

The principle of jet grinding is that solids particles are accelerated by high-speed gas flow and fragmented due to multiple particle-particle collisions in interacting gasparticle jet. The development of jet grinding technology mainly includes the development of basic theory of jet grinding and its device, and the former is the foundation of the latter. According to the principle of jet grinding, the grind energy of particles comes from high-speed gas. Therefore, the analysis on the particle acceleration progress is a key point in the design of jet grinding device.

Whether the particles in the gas flow can be effectively accelerated and collided at its maximum velocity is the important condition to improve the efficiency of jet grinding. Researches of particles' acceleration in nozzles have been reported in some literatures [1-3], but there have not any reports about particles' acceleration in fluidized bed. If the accelerated distance is too far, when the particle is accelerated to the maximum velocity, it will be affected by the gas flow and solids, and slow down; if the accelerated distance is too close, particle can not be accelerated enough. So, to determine the optimal injection distance is very important to improve the efficiency of jet grinding. Reference $[4,5]$ pointed out that it was a preferable choice that the accelerated distance of nozzles was as 10 - 20 times as the dimensionless distance, but the result is too wide. On the other hand, although many papers on the analysis of particle motion do not consider the influence of gravity, for tough particle, the gravity, as well as the angle of inclination of the nozzle, has great

${ }^{*}$ Corresponding author. influence on the efficiency of the fluidized bed.

To investigate the particle motion in the high-speed gas flow better, this paper establishes some different mathematical models to describe particle acceleration with and without considering gravity, respectively. Meanwhile, numerical simulations show the trend of the velocity of the particle under different conditions and the relationship between the maximum particle velocity and its diameter size.

\section{Particle Acceleration Model without Gravity}

\subsection{Mathematical Model}

Without considering gravity, the equation of a single particle motion [6] is as follows

$$
\frac{d u_{s}}{d t}=\frac{0.75 C_{d} \rho}{\rho_{s} d_{s}}\left(u(t)-u_{s}\right)^{2}
$$

where $u_{s}$ is the velocity of particle, $u(t)$ is the velocity of gas, $\rho$ is the gas density, $\rho_{s}$ is the particle density, $d_{s}$ is the particle diameter size and $C_{d}$ is the particle drag force coefficient.

Equation (1) indicates the velocity of particle $U_{s}$ is a function in $t$. By transformation, it can be rewritten to an equation about the particle velocity and the jet distance,

$$
u_{s} \frac{d u_{s}}{d \bar{x}}=\frac{0.75 C_{d} \rho d_{e}}{\rho_{s} d_{s}}\left(u(\bar{x})-u_{s}\right)^{2},
$$

Where $\bar{X}$ is the dimensionless distance which equals to the ratio of the distance of jet stream section to the nozzle 
outlet and the diameter of the nozzle outlet, $u(\bar{x})$ is the velocity of gas in the dimensionless distance and $d_{e}$ is the diameter size of the nozzle outlet.

Depending on the injecting high-speed gas flow, most particles can be accelerated in axis velocity area, but the existing problem is that some particles are accelerated at the edges of area (see Figure 1). Thus, except for the axis velocity of gas, we should also consider the mean velocity of quality of gas. Only in this way, can we scientifically evaluate the acceleration effect.

Assume that the axis velocity of gas changes in accordance with the jet.

In initial part, $\bar{x} \leq 4.4$

$$
\begin{aligned}
& u(\bar{x})=u, \quad(\text { Axis velocity }), \\
& u(\bar{x})=\frac{u}{1+1.52 a \bar{x}+5.28 a^{2} \bar{x}^{2}}
\end{aligned}
$$

(Mean velocity of quality)

where $a=0.076$ is the coefficient of turbulence.

In main part,

$$
\begin{aligned}
& u(\bar{x})=\frac{6.3 u}{\bar{x}+1.93} \text { (Axis velocity), } \\
& u(\bar{x})=\frac{3 u}{\bar{x}+1.93} \\
& (\text { Mean velocity of quality). }
\end{aligned}
$$

\subsection{Influence of the Axis Velocity on Particle Acceleration}

Using air as the working medium, and by FLUENT software we get the testing data of gas flow field in the 30 times dimensionless distance, that is, array $[\bar{x}, y]$, where $\bar{x}$ is the dimensionless distance, $y$ is the parameter group including gas velocity, gas density and viscosity.

Next, let $d_{e}=16 \mathrm{~mm}$, and $\rho_{s}=2650 \mathrm{~kg} / \mathrm{m}^{3}$ and $\rho_{\mathrm{s}}=7950 \mathrm{~kg} / \mathrm{m}^{3}$, respectively.
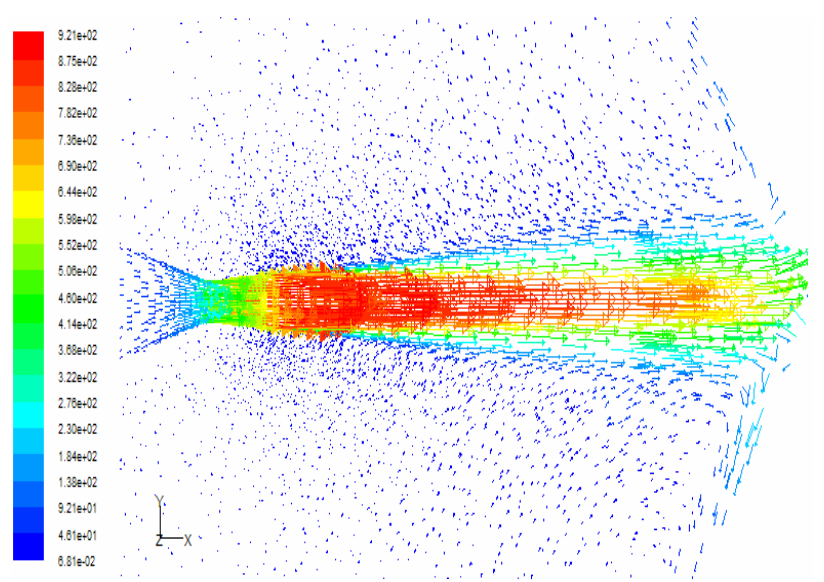

Figure 1. The velocity vectors of gas in fluidized bed jet grinding.
We also take $d_{e}=50 \mu \mathrm{m}, 150 \mu \mathrm{m}, 250 \mu \mathrm{m}, 500 \mu \mathrm{m}$ and $750 \mu \mathrm{m}$, respectively, and employ equation (3) and (5) to compute the velocity of gas. After discretization of equation (2) and by Newton iteration, we get the relationship between the particle acceleration and the dimensionless distance (see Figure 2 and Figure 3).

It is clearly observed from Figure $\mathbf{2}$ and Figure $\mathbf{3}$ that, when the curve of particle acceleration crosses the curve of the velocity of gas, particle reaches its maximum velocity, and the corresponding dimensionless distance is the optimal distance for nozzle jet.

In general, the smaller the particle diameter size is, the easier it is to be accelerated. When the particle density is low, the smaller the particle diameter size is, the more obvious the acceleration of velocity is; but if the density is high, the speed change will not be obvious no matter the size of the particle is big or small.

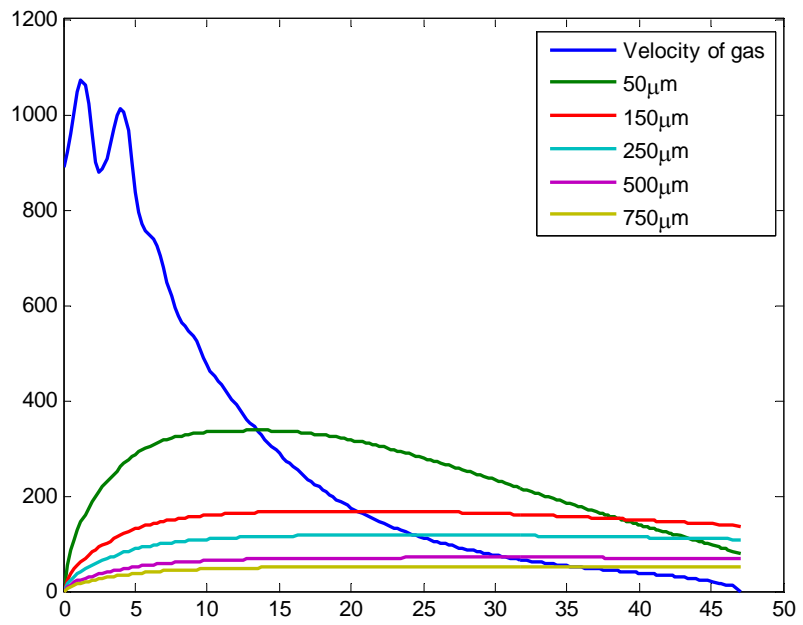

Figure 2. The velocity of particle and the axis velocity of gas with $\rho_{s}=2650 \mathrm{~kg} / \mathrm{m}^{3}$.

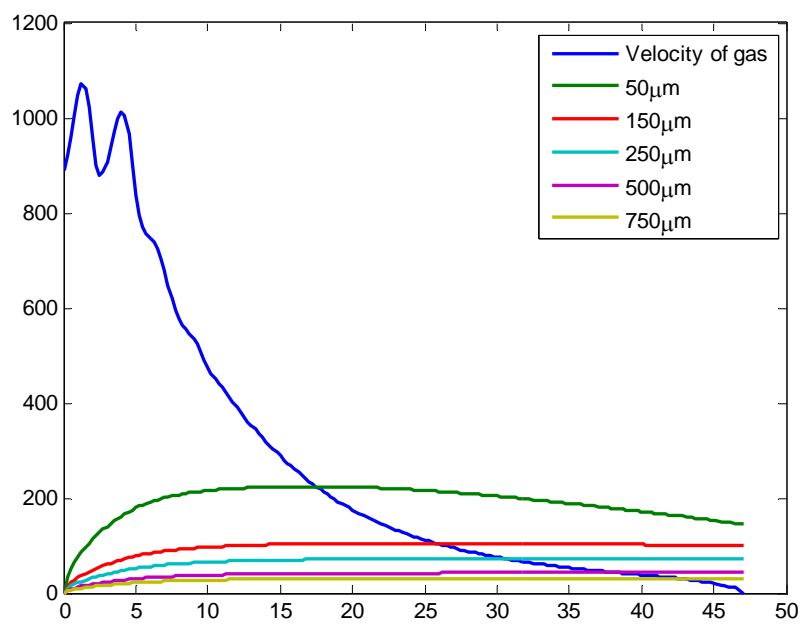

Figure 3. The velocity of particle and the axis velocity of gas with $\rho_{s}=7950 \mathrm{~kg} / \mathrm{m}^{3}$. 
For $\rho_{s}=2650 \mathrm{~kg} / \mathrm{m}^{3}$, when particle diameter is $d_{s}$ $=50 \mu \mathrm{m}$, the $0-10$ times dimensionless distance is the particle's rapidly accelerating phase, the 10 - 20 times dimensionless distance is its slow rise and slow reduction phase, and the 20 - 30 times dimensionless distance is particle velocity's sharp decline phase. At 14 times dimensionless distance, particle reaches its maximum velocity of $378 \mathrm{~m} / \mathrm{s}$.

At the same time, Figure 4 also shows the relationship between the particle maximum velocity and the particle diameter size with particle density $\rho_{s}=5300 \mathrm{~kg} / \mathrm{m}^{3}$.

\subsection{Influence of the Mean Velocity of Quality on Particle Acceleration}

Parameters $\rho_{s}, d_{s}, d_{e}$ and $C_{d}$ are taken as in section 2.2. Here we employ equation (4) and (6) to compute the velocity of gas. By the same computation method, we get the relationship between the particle acceleration and the dimensionless distance (see Figure 5 and Figure 6).

From the above figures, we can see that the quality mean velocity of gas flow decays faster than the axis velocity of gas. In 0 - 25 times dimensionless distance, the difference between the quality mean velocity and axis velocity increases along with the increase of dimensionless distance, because the gas flow is more and more divergent with the increase of the dimensionless distance.

In 25 - 30 times, the situation conversed. The main reason is that when gas collision happens in the grinding center, the anti-shock wave will be formed, and then the axis velocity of gas will rapidly decay.

The trend of the particle velocity gets something similar to the abovementioned result, but the concrete numerical result is slightly different. For $\rho_{s}=2650 \mathrm{~kg} / \mathrm{m}^{3}$, when particle diameter is $d_{s}=50 \mu \mathrm{m}$, the 0 - 8 times dimensionless distance is the particle's rapidly accelerating

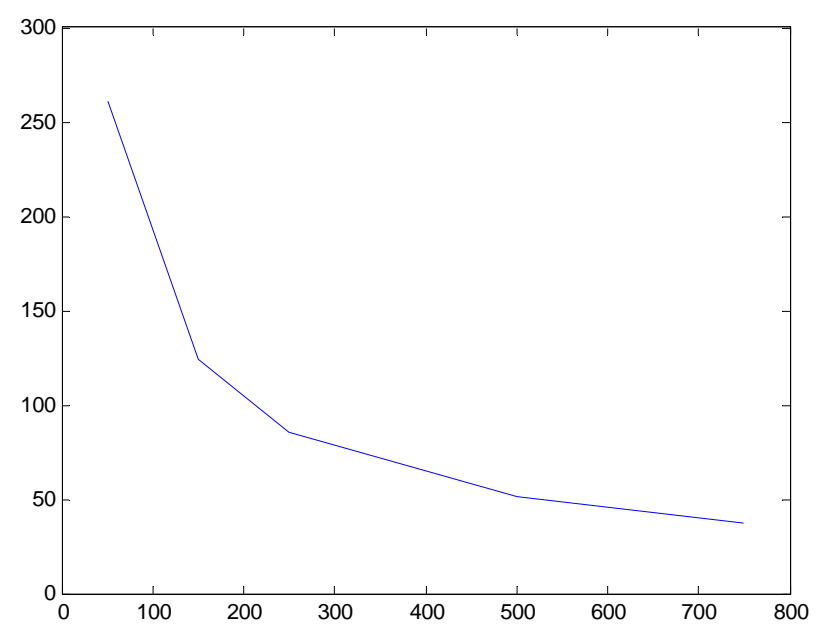

Figure 4. The relationship between the particle maximum velocity and the particle diameter size with particle density $\rho_{s}=5300 \mathrm{~kg} / \mathrm{m}^{3}$.

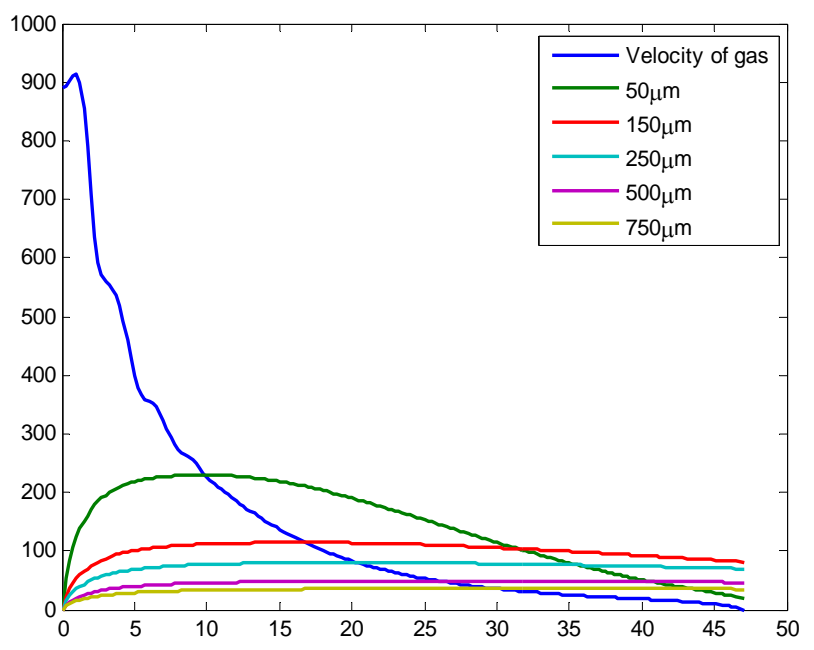

Figure 5. The velocity of particle and the mean velocity of quality of gas with $\rho_{s}=2650 \mathrm{~kg} / \mathrm{m}^{3}$.

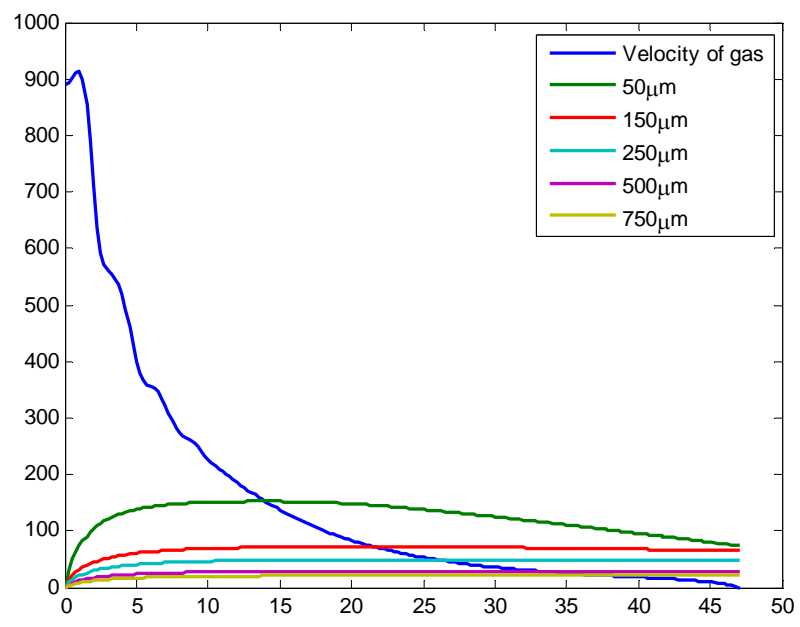

Figure 6. The velocity of particle and the mean velocity of quality of gas with $\rho_{s}=7950 \mathrm{~kg} / \mathrm{m}^{3}$.

phase, the 8 - 17 times dimensionless distance is its slow rise and slow reduction phase, and the 17 - 30 times dimensionless distance is particle velocity's sharp decline phase. At 10 times dimensionless distance, particle reaches its maximum velocity of $223 \mathrm{~m} / \mathrm{s}$.

Similarly, Figure 7 also shows the changing relationship between the particle maximum velocity and the particle diameter size with particle density $\rho_{s}=5300 \mathrm{~kg} / \mathrm{m}^{3}$.

\section{Particle Acceleration Model with Gravity}

\subsection{Mathematical Model}

With considering the gravity of the particle, equation (2) can be revised as follows,

$$
u_{s x} \frac{d u_{s X}}{d \bar{x}}=\frac{0.75 C_{d} \rho d_{e}}{\rho_{s} d_{s}}\left(u(\bar{x})-u_{s x}\right)^{2},
$$




$$
\frac{d u_{s y}}{d t}=g,
$$

where $u_{s x}, u_{s y}$ mean the horizontal and vertical velocity of the particle, respectively. The particle velocity $u_{s}$ can be obtained by

$$
u_{s}=\sqrt{u_{s x}^{2}+u_{s y}^{2}},
$$

Because the direction of movement is no longer horizontal, the corresponding angle of inclination of the nozzle outlet can approximately represent as:

$$
\theta=\arctan l_{y} / l_{x}
$$

where $l_{x}, l_{y}$ mean the horizontal and vertical displacement of the particle at its maximum speed, respectively.

\subsection{Influence of the Axis Velocity on Particle Acceleration}

Here we only take $\rho_{s}=2650 \mathrm{~kg} / \mathrm{m}^{3}$ to discuss the influence of the axis velocity. Other parameters and axis velocity are taken as in section 2.2. After discretization of equation (7) and (8) by Newton iteration, we also get the relationship between the particle acceleration and the dimensionless distance with gravity (see Figure 8).

It can be perceived from the Figure 8 that, the change of particle velocity does not have much impact, with considering the gravity. The main reason for this is that the particle density is not high, and particle size is small, which makes the effect of gravity on particle acceleration process not so obvious. However, gravity will produce the vertical direction of the displacement. To achieve better crushing impact, we must adjust nozzle angle according to the influence of gravity. For example, Figure 9 shows Particle trajectory with $\rho_{s}=2650 \mathrm{~kg} / \mathrm{m}^{3}$ and $d_{s}=750 \mu \mathrm{m}$.

By equation (10), we can get

$$
l_{y}=0.0011 \mathrm{~m}, l_{x}=0.5680 \mathrm{~m}, \theta=0.1^{\circ} .
$$

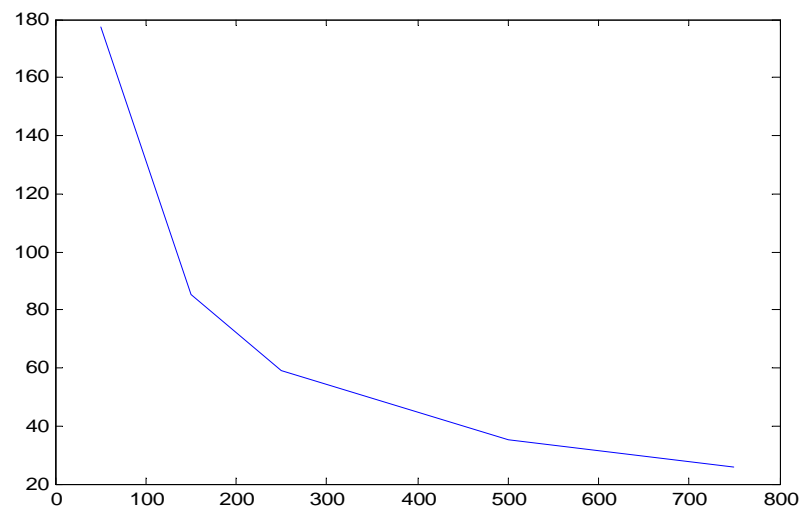

Figure 7. The relationship between the particle maximum velocity and the particle diameter size with particle density $\rho_{s}=5300 \mathrm{~kg} / \mathrm{m}^{3}$.

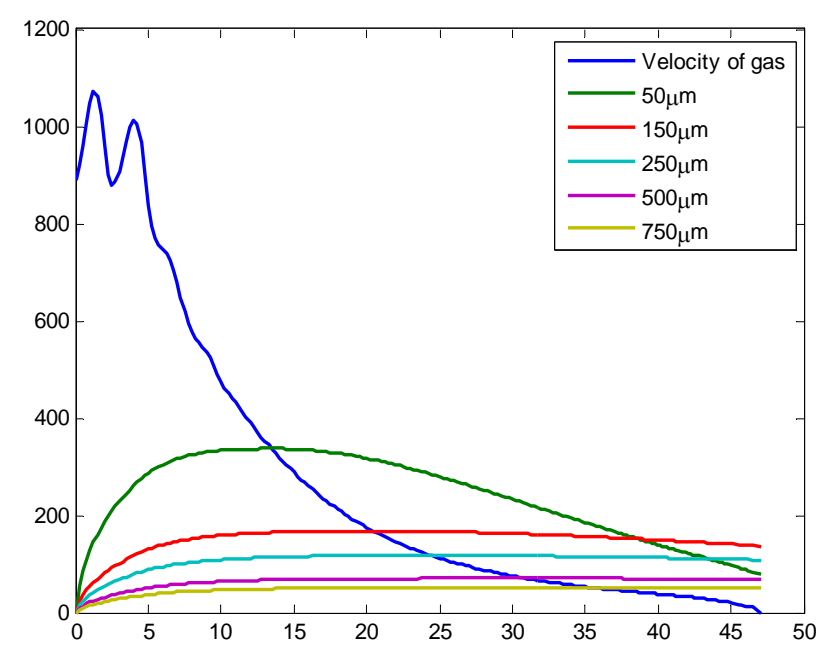

Figure 8. The velocity of particle and the axis velocity of gas with $\rho_{s}=2650 \mathrm{~kg} / \mathrm{m}^{3}$ under considering gravity.

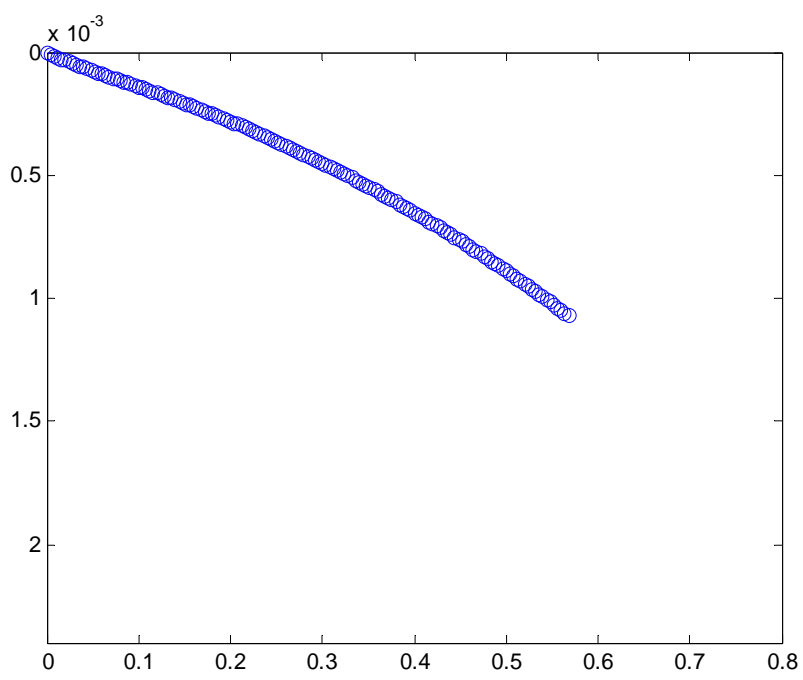

Figure 9. Particle trajectory with $\rho_{s}=2650 \mathrm{~kg} / \mathrm{m}^{3}$ and $d_{s}$ $=750 \mu \mathrm{m}$.

If high-density and coarse particle for parameters are taken, angle may correspondingly increase. To verify this fact, we also present the result in Figure 10 which shows particle trajectory with $\rho_{s}=7950 \mathrm{~kg} / \mathrm{m}^{3}$ and $d_{s}=1000$ $\mu \mathrm{m}$.

We get (Figure 11)

$$
l_{y}=0.0068 \mathrm{~m}, l_{x}=0.7101 \mathrm{~m}, \theta=0.5489^{\circ} .
$$

\subsection{Influence of the Mean Velocity of Quality on Particle Acceleration}

In this section, we also investigate the trend of velocity of the particle by the quality mean velocity of gas. The corresponding results of these simulations are given as follows, The reason is similar to the statement in section 
2.3, and we do not explain it here repeatedly.

From Figure 12, we have

$$
l_{y}=0.0014 \mathrm{~m}, l_{x}=0.4840 \mathrm{~m}, \quad \theta=0.16^{\circ} .
$$

Accordingly, (Figure 13)

$$
l_{y}=0.0109 \mathrm{~m}, l_{x}=0.6667 \mathrm{~m}, \theta=0.9^{\circ} .
$$

From the numerical simulation in section 3.2 and 3.3, it can be seen that the influence of gravity in the process of particle acceleration is not obvious. While aimed at high-density and coarse particle, the influence of gravity needs to be considered.

\section{Conclusions}

This paper mainly focuses on acceleration of the particle in the process of jet grinding. On the one hand, based on a basic particle motion model, the relationships of particle velocity with gas velocity, particle density and particle diameter size are obtained through numerical simulations.

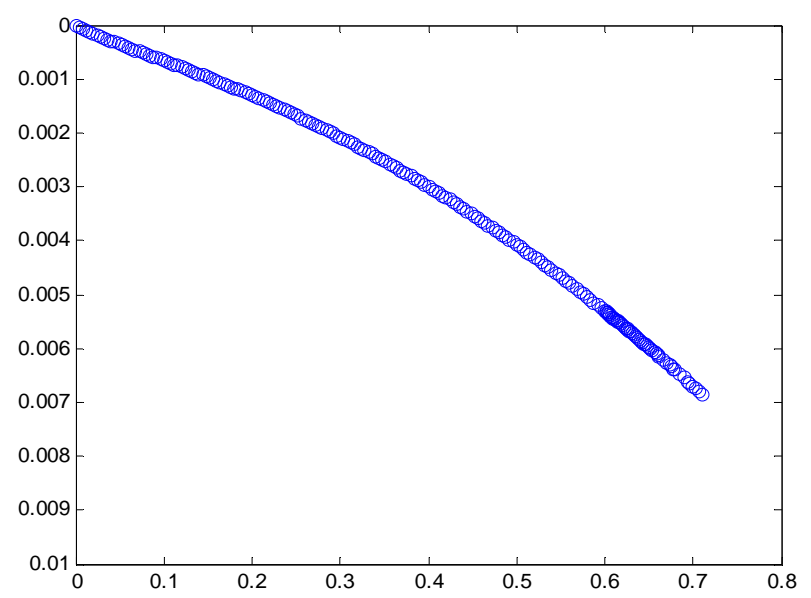

Figure10. Particle trajectory with $\rho_{s}=7950 \mathrm{~kg} / \mathrm{m}^{3} d_{s}=$ $1000 \mu \mathrm{m}$.

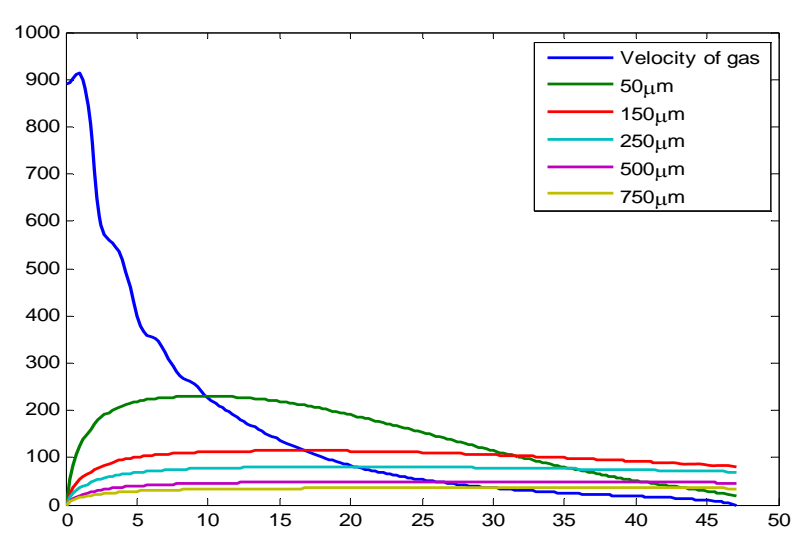

Figure 11. The velocity of particle and the mean velocity of quality of gas with $\rho_{s}=2650 \mathrm{~kg} / \mathrm{m}^{3}$ under considering gravity.

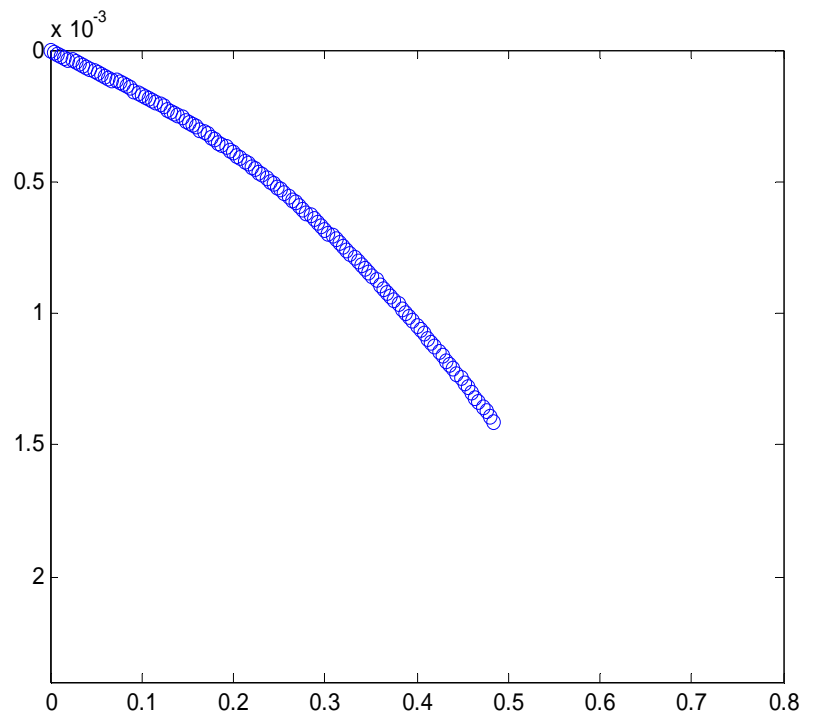

Figure 12. Particle trajectory with $\rho_{s}=2650 \mathrm{~kg} / \mathrm{m}^{3}$ and $d_{s}=750 \mu \mathrm{m}$.

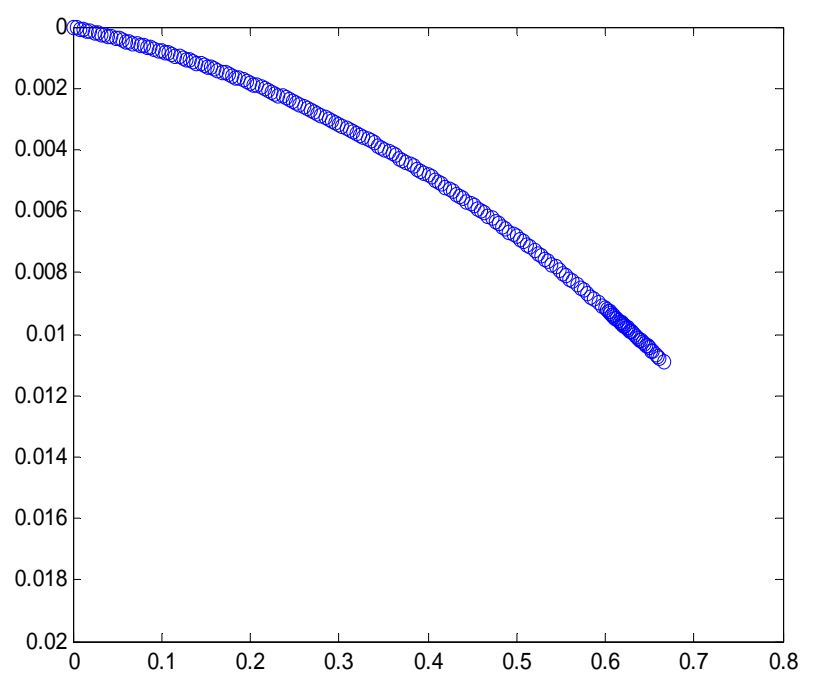

Figure 13. Particle trajectory with $\rho_{s}=7950 \mathrm{~kg} / \mathrm{m}^{3}$ and $d_{s}=1000 \mu \mathrm{m}$.

On the other hand, the mathematical model of particle acceleration under the influence of gravity is established and its numerical simulation is also carried out. Theoretical analysis will have great guidance to the improvement of the fluidized bed jet grinding technology.

\section{Acknowledgements}

This work is supported by the Doctoral Program Research Fund of Southwest University of Science and Technology (No.11zx7129) and the National Science and Technology Support Program (No. 2011BAA04B04). 


\section{REFERENCES}

[1] W. Gregor and K. Schönert, "The Efficiency of the Particle Acceleration in a Jet Pipe,” Powder Technology, Vol. 34, No. 1, 1983, pp. 81-86. doi:10.1016/0032-5910(83)87031-4

[2] D. Eskin, S. Voropayev and O. Vasilkov, "Simulation of Jet Milling,” Powder Technology, Vol. 105, No. 1-3, 1999, pp. 257-265. doi:10.1016/S0032-5910(99)00146-1

[3] D. Eskin and S. Voropayev, "Engineering Estimations of Opposed Jet Milling Efficiency,” Minerals Engineering, Vol. 14, No. 10, 2001, pp.1161-1175.
doi:10.1016/S0892-6875(01)00134-0

[4] S. M. Tasirin and D. Geldart, "Experimental Investigation on Fluidized Bed Jet Grinding,” Powder Technology, Vol. 105, No. 1-3, 1999, pp. 333-341. doi:10.1016/S0032-5910(99)00156-4

[5] J. Kolacz, "Process Efficiency Aspects for Jet Mill Plant Operation,” Minerals Engineering, Vol. 17, No. 11-12, 2004, pp. 1293-1296.

doi:10.1016/j.mineng.2004.07.004

[6] H. Chen, "Study and Application of the Fluidized Bed Jet Grinding and Classifying Technology," Ph. D. Thesis, Sichuan University, Chengdu, 2007. 